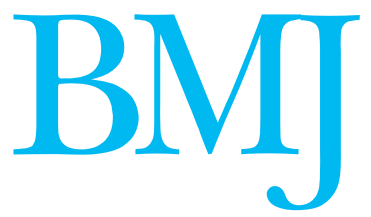

\title{
The polypill and cardiovascular disease
}

\author{
May be appropriate for secondary, but perhaps not for primary prevention
}

Papers p 1059
$\mathrm{T}$ The prevention of cardiovascular disease with drug therapy is well known. Randomised controlled trials and meta-analyses of trials of lipid and blood pressure lowering and antiplatelet therapy have established their efficacy in the prevention of cardiovascular diseases. Wald and Law have proposed that these three treatments, along with folic acid, be combined into a "polypill." They propose a combined strategy for primary and secondary preventiontargeting all people with pre-existing cardiovascular disease (secondary prevention) but more controversially, targeting all adults aged over 55 (primary prevention) as well. The underlying assumption concerning the efficacy of this strategy is that the six individual ingredients of the polypill (thiazide diuretic, angiotensin converting enzyme inhibitor, $\beta$ blocker, statin, aspirin, and folic acid) when combined together have synergistic treatment effects - calculated by multiplying the relative risk reductions on each class of treatment. Their polypill strategy has generated worldwide interest, with some critics questioning this underlying multiplicative assumption as being too optimistic.

For these reasons, the paper by Hippisley-Cox and Coupland in this issue ( $p$ 1059), examining the individual and combined effects of three of the polypill ingredients-statins, aspirin, and blood pressure lowering drugs is timely. ${ }^{2}$ Their analysis provides support for the synergic action of the polypill in the context of secondary prevention of coronary heart disease. Their analysis of 11330 patients with coronary heart disease shows that all cause mortality is lower in those patients taking drug combinations-two or three drugs when compared with those taking single agents. These findings are consistent with a previous study that showed that a combination of two drugs, aspirin and pravastatin, is superior to either drug alone in the secondary prevention of cardiovascular disease. ${ }^{3}$ A further study of dispensed prescribing in the secondary prevention of coronary heart disease in 4892 patients in Tayside, Scotland, also shows that patients taking an additional cardiovascular drug experience fewer cardiovascular events than patients taking statins alone, but that this synergistic effect was not sustained when two additional drugs were taken: hazard ratios for combinations of two and three drugs were the same. ${ }^{4}$ Overall these studies provide support for the synergistic effects of two, but not three or four, drug combinations in secondary prevention. However, these studies are non-randomised comparisons of outcomes and are therefore prone to confounding by severity of disease and other factors.
In the context of primary prevention many uncertainties remain. Recent evidence concerning the differential effect of aspirin in women compared with men is emerging. While the efficacy of aspirin in men is established, ${ }^{5}$ the recently completed women's health study, of low dose aspirin (100 mg every other day) compared with placebo, did not produce a reduction in all cause mortality or fatal and non-fatal myocardial infarction. ${ }^{6}$ Although observational evidence favours a possible causal association between raised plasma homocysteine concentrations and cardiovascular disease ${ }^{7}$ this association has been described as modest; a $25 \%$ reduction in usual homocysteine concentrations is associated with a $11 \%$ lower risk of coronary heart disease and a $19 \%$ lower risk of stroke. ${ }^{8}$ We have growing evidence from approaches using mendelian randomisation that the expected effects of the folic acid component may not be confirmed. ${ }^{9}$ Furthermore, a recent randomised trial of $2.5 \mathrm{mg}$ /day of folic acid (the proposed polypill dosage is $0.8 \mathrm{mg} /$ day) was not associated with a reduction in the combined trial end point of stroke, coronary events, and death in patients who has previously had a cerebral infarction. ${ }^{10}$ In the light of conflicting evidence between observational and randomised controlled studies concerning the benefits of antioxidant vitamins and prevention of cardiovascular disease, caution is needed before extrapolating the benefits from observational studies to benefit from treatment with the folic acid component of the polypill. ${ }^{11}$

Other concerns about the primary prevention strategy of the polypill relate to its non-specific scattershot primary prevention approach, which would expose people at lower risk to lifelong treatment, with attendant medicalising of the population. ${ }^{12}$ Cost effectiveness analyses that estimated the impact of cardiovascular risk in the strategy to treat hypertension have shown that treating individuals at high risk is highly cost effective, irrespective of age or sex. However, individuals at lower risk (who represent most of the adult population) are much more expensive to treat in terms of gain in quality adjusted life years. ${ }^{13}$ Furthermore, the preference of patients has a strong bearing on the cost effectiveness of treatment in these low risk individuals. ${ }^{13}$

The underlying tenet of the polypill- that combination therapy is better than monotherapy-may well be correct, particularly with regard to secondary prevention of cardiovascular disease. Hippisley-Cox and Coupland's paper goes some way in providing data concerning the effects of combined treatment in 
secondary prevention of coronary heart disease. In terms of primary prevention, development and testing of combination pills aimed at reducing more than one risk factor seems entirely logical, particularly in the context of assessment of global cardiovascular risk. Funding bodies and the NHS need to support the necessary trials and cost effectiveness studies to further examine the polypill strategy in comparison with nonpharmacological alternatives.

Tom Fahey professor of primary health care

Tayside Centre for General Practice, University of Dundee, Dundee DD2 4AD

Peter Brindle Welcome training fellow in health services research

Shah Ebrahim professor in the epidemiology of ageing

Department of Social Medicine, University of Bristol, Bristol BS8 2PR

Competing interests: None declared.

1 Wald NJ, Law MR. A strategy to reduce cardiovascular disease by more than $80 \%$. BMI 2003;326:1419.

2 Hippisley-Cox J, Coupland C. Effect of combinations of drugs on all cause mortality in patients with ischaemic heart disease: nested case-control analysis. BMJ 2005:330:1059-63.

3 Hennekens CH, Sacks FM, Tonkin A, Jukema J, Byington R, Pitt B, et al. Additive benefits of pravastatin and aspirin to decrease risks of cardiovascular disease. Arch Intern Med 2003;164:40-4.
4 Wei L, Ebrahim S, Bartlett C, Davey PD, Sullivan FM, MacDonald TM. Statin use in the secondary prevention of coronary heart disease in primary care: cohort study and comparison of inclusion and outcome with patients in randomised trials. BMJ 2005;330:821.

5 Eidelman R, Herbert P, Weisman S, Hennekens CH. An update on aspirin in the primary prevention of cardiovascular disease. Arch Intern Med 2005; 163:2006-10.

6 Ridker PM, Cook N, Lee I, Gordon D, Gaziano JM, Manson JE, et al. A randomised trial of low-dose aspirin in the primary prevention of cardiovascular disease in women. N Engl J Med 2005;352:1293-304.

7 Wald DS, Law M, Morris JK. Homocysteine and cardiovascular disease evidence on causality from a meta-analysis. BMJ 2002;325:1202-6.

8 The Homocysteine Studies Collaboration. Homocysteine and risk of ischaemic heart disease and stroke. JAMA 2002;288:2015-22.

9 Frederiksen J, Juul K, Grande P, Jensen GB, Schroeder TV, Tybjaerg-Hansen A, et al.. Methylenetetrahydrofolate reductase polymorphism (C677T), hyperhomocysteinemia, and risk of ischemic cardiovascular disease and venous thromboembolism: prospective and casecontrol studies from the Copenhagen city heart study. Blood 2004;104:3046-51.

10 Toole J, Malinow MCL, Spence J, Pettigrew L, Howard V, Sides E, et al. Lowering homocysteine in patients with ischemic stroke to prevent recurrent stroke, myocardial infarction and death. The vitamin intervention for stroke prevention (VISP) randomized controlled trial. JAMA 2004;291:565-75.

11 Lawlor DA, Smith GD, Bruckdorfer K, Kundu D, Ebrahim S. Those confounded vitamins: what can we learn from the differences between observational versus randomised trial evidence? Lancet 2004;363:1727.

12 Mulrow CD, Kussmaul W. The middle-aged and older American: wrong prototype for a preventive polypill? Ann Inter Med 2005; 142:467-8.

13 Montgomery AA, Fahey T, Harding J, Ben-Shlomo Y. The influence of absolute cardiovascular risk, patient utilities and costs on the decision to treat hypertension: a Markov decision analysis. $J$ Hypertens 2003;21:1753-9.

\section{Pharmacogenetics and ethnically targeted therapies}

\section{New drug BiDil marks the return of biology to the debate about race and ethnicity}

I

$\mathrm{n}$ modern conceptions of race and ethnicity, biology has been relegated to a minor underlying factor. ${ }^{1}$ Instead, these concepts have been cast as largely social constructions. ${ }^{2}$ For example, race traditionally distinguishes between groups according to a mixture of physical characteristics (including skin colour), which reflect ancestry and hence biology. A modern conception of race would place the emphasis on a common social and political heritage. Similarly, ethnicity puts emphasis on distinguishing between groups by using a mixture of cultural factors, including language and religion. ${ }^{2}$ Recent developments in pharmacogenetics, however, renew the historical emphasis on biology in concepts of race and ethnicity. Pharmacogeneticists examine whether different responses to drug treatment may be attributable to genetic differences. They are focusing on race and ethnicity as a means to this end. A recent international conference, the 8th world congress on clinical pharmacology and therapeutics, in Brisbane, Australia, had an afternoon on ethnopharmacology, showing how seriously this new subject is being taken.

In the middle of this resurgence of the role of biology in concepts of race and ethnicity comes BiDil, a new drug treatment for heart failure tested solely in one particular racial group. In 2001 NitroMed began the African-American heart failure trial (A-HeFT), the first heart failure trial conducted exclusively in African-American patients, claiming that "observed racial disparities in mortality and therapeutic response rates in Black heart failure patients may be due in part to ethnic differences in the underlying pathophysiology of heart failure." ${ }^{45}$ The study found that BiDil (a fixed dose of isosorbide dinitrate and hydralazine, designed to restore low or depleted nitric oxide concentrations to the blood) combined with standard therapy for heart failure reduced mortality by $43 \%$ among black patients. ${ }^{5}$ Hailed by the media as the first ethnic drug, BiDil is reported to be on the way to becoming the first drug approved by the US Food and Drug Administration to treat heart failure in African-American patients only. ${ }^{6}$

The major implication of BiDil is that differential responses to treatment between racial groups, defined by using ostensibly social categories (here, patients self reported to be African-Americans), are attributed primarily to genetic differences. If this is shown to be true it will undermine a postwar consensus emphasising the social construction of race and ethnicity. Largely social categories of race and ethnicity may be useful indicators of genetic variations because they are at least partly based on biological characteristics. If everyone were the same physically-skin colour and so on-racial and ethnic categories would not exist. This raises fundamental and controversial questions. Do important genetic differences exist between ethnic and racial groups as defined by current classifications? If they do, how good are current racial labels as an indicator of these genetic differences? Should such classifications be used in this way? If so, will race science see a resurgence? ${ }^{1}$

Many researchers and policy makers argue against the use of racial or ethnic categories in medicine, saying that classifying people according to race and ethnicity reinforces existing social divisions in society or leads to discriminatory practices. ${ }^{7}$ Others cite 